\title{
Van Kedilerinde Skeleton Manus'un Bilgisayarlı Tomografi ile Üç Boyutlu Olarak Incelenmesi*
}

\author{
Osman YILMAZ ${ }^{1 \mathrm{a} \otimes}$, Zafer SOYGÜDER ${ }^{1 \mathrm{~b}}$, Alpaslan YAVUZ $^{2 \mathrm{c}}$
}

1. Van Yüzüncü Yıl Üniversitesi, Veteriner Fakültesi, Anatomi Anabilim Dalı, Van, TÜRKiYE. 2. Van Yüzüncü Yıl Üniversitesi, Tıp Fakültesi, Radyoloji Anabilim Dalı, Van, TÜRKiYE. ORCID: 0000-0003-2013-9213 ${ }^{\mathrm{a}}$, 0000-0002-0489-5062 ${ }^{\mathrm{b}}, 0000-0002-8796-988 \mathrm{X}^{\mathrm{c}}$

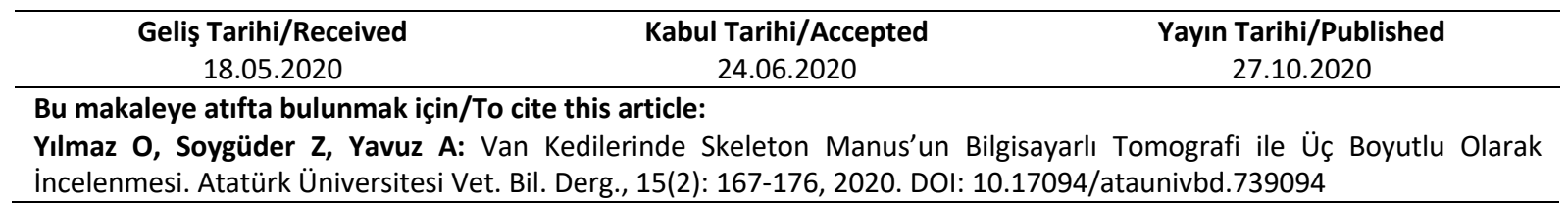

Öz: Bu çalışma, Van kedilerinde skeleton manus (ossa carpi, ossa metacarpalia I-V ve ossa digitorum manus)'un bilgisayarlı tomografi (BT) ile üç boyutlu (3B) rekonstrüksiyonunu yapmak, osteometrik ölçümlerini belirlemek ve bu ölçüm değerlerinin erkek ve dişi kediler arasındaki biyometrik farklılıklarının belirlenmesi amacıyla yapıldı. Çalışmada 16 adet (8 dişi, 8 erkek) yetişkin Van kedisi kullanıldı. Xylazin-ketamin kombinasyonu ile anesteziye alınan kedilerin BT ile taranarak görüntüleri elde edildi. Daha sonra bu axial imajlar DICOM formatında işlenmek üzere iş istasyonuna aktarıldı ve 3B modelleme programı olan Syngo CT kullanılarak rekonstrüksiyon işlemi yapıldı. Üç boyutlu hale getirilen rekonstrüksiyon görüntülerindeki kemikler anatomik özellikleri bakımından incelendi. Bu görüntülerden volümetrik ve morfometrik ölçüm değerleri elde edilerek, istatistiki olarak analizi yapıldı. Morfometrik analiz sonuçlarına bakıldığında, ossa carpi, ossa metacarpalia I-V ve ossa digitorum manus'un ölçüm değerleri bakımından erkek ve dişi Van kedilerinde istatistiksel farklılıklar olduğu görüldü $(P<0.05)$. Ossa carpi, ossa metacarpalia I-V ve ossa digitorum manus'un volümetrik ölçüm değerlerinin ise erkek kedilerde dişi kedilere göre istatistiki olarak önemli seviyede daha fazla olduğu gözlendi $(P<0.05)$. Sonuç olarak, Van kedilerinde skeleton manus volümetrik, morfometrik ve morfolojik olarak değerlendirildi. Bu kemiklerin osteometrik ölçüm değerlerinin istatistiksel olarak cinsiyetler arasındaki biyometrik farklılıkları belirlendi.

Anahtar Kelimeler: Bilgisayarlı tomografi, Üç boyutlu rekonstrüksiyon, Skeleton manus, Van kedisi.

\section{Three-Dimensional Investigation by Computed Tomography of The Skeleton Manus in Van Cats}

Abstract: This study aimed to obtain the three-dimensional (3D) reconstruction by computed tomography (CT) and the osteometric measurements of the skeleton manus (ossa carpi, ossa metacarpalia I-V ve ossa digitorum manus) in adults Van cats, and to determine the biometric differences between these measurement values in male and female cats. In the study, 16 adult Van cats ( 8 females, 8 males) were used. The cats that were anesthetized with the xylazine-ketamine combination were scanned by CT device, and images of skeleton manus were obtained. These axial images were transferred to the workstation for processing in DICOM format and then reconstruction was performed using Syngo CT, a 3D reconstruction program. The bones in three-dimensional reconstruction images were evaluated for their anatomical features. Volumetric and morphometric measurement values were obtained from these images and statistical analyzes were done. In the results of morphometric analyses, statistically significant differences $(P<0.05)$ were seen between measured values of the carpal bones, metacarpal I-V bones and phalanges of the digits in male and female Van cats. The volumetric measurement values of the carpal bones, metacarpal I-V bones and phalanges of the digits were statistically significantly greater in male cats than in female cats $(P<0.05)$. In conclusion, the carpal bones, metacarpal I-V bones and phalanges of the digits were evaluated volumetric, morphometric, and morphologically in Van cats. The biometric differences between the osteometric measurement values of these bones in both sexes were examined statistically.

Keywords: Computed tomography, Skeleton manus, Three-dimensional reconstruction, Van cat.

区 Osman YıImaz

Van Yüzüncü Yıl Üniversitesi, Veteriner Fakültesi, Anatomi Anabilim Dalı, TÜRKiYE.

e-posta: osman_40_5@hotmail.com

* Bu çalışma, birinci yazarın “Van Kedilerinde Ön Bacak İskeletinin Bilgisayarlı Tomografi ile Üç Boyutlu Olarak İncelenmesi” isimli doktora tezinin bir bölümünden oluşmaktadır ve Van Yüzüncü Yıl Üniversitesi Bilimsel Araştırma Projeleri Koordinasyon Birimi tarafından TDK2017-5905 proje numarası ile desteklenmiştir. 


\section{Giriş}

$\mathrm{V}$ an yöresinde yetişen ve ismini buradan alan Van kedileri, farklı göz rengi, üçgen şeklinde kafa yapısı, uzun vücut yapısı, vücutlarında değişik boylarda (kısa, orta, uzun) ve yumuşak tüyler (genellikle fildişi renginde) bulundurması, bol tüylü kuyruğu, temizliği, yüzmeyi sevmesi, zekiliği ve üstün öğrenme yeteneğiyle dikkat çekmektedir (1).

Skeleton manus (autopodium), proximal'den distal'e doğru, ossa carpi (basipodium), ossa metacarpalia (metapodium) ve ossa digitorum manus (acropodium)'tan oluşur (2-4). Skeleton manus'ta memeli hayvanların yaşam koşullarına uygun olarak ve yere basış şekillerine göre bazı özel modifikasyonlar görülür. Memeli hayvanlar yere basış şekillerine göre, plantigrad (tabanlarıyla yere basanlar; insan, maymun, ayı gibi), digitigrad (parmakları ile yere basanlar; carnivorlar gibi) ve unguligrad (tırnakları ile yere basanlar; equus, ruminant ve sus gibi) memeliler olmak üzere 3 gruba ayrılırlar. Plantigrad ve digitigrad'ların ön ayağında normal gelişmesine uygun olarak bütün parmaklar (beş adet) mevcuttur. Unguligrad'lardan equus'ta bir parmak (3. parmak), ruminant'ta iki parmak (3. ve 4. parmaklar), sus'ta dört parmak (2., 3., 4., ve 5. parmaklar) vardır (5).

Son zamanlarda bilgisayarlı tomografi gibi medikal görüntüleme yöntemleri ve üç boyutlu modellemeler sayesinde anatomi öğretiminde önemli değişiklikler meydana gelmiştir $(6,7)$. Özellikle kedi, köpek gibi küçük pet hayvanlarında bilgisayarlı tomografi ve üç boyutlu rekonstrüksiyon programları iskelet sistemindeki anatomik yapıların morfolojik, morfometrik, volümetrik ve patolojik olarak değerlendirilmesinde oldukça yaygın bir şekilde kullanılmaktadır $(8,9)$.

Bu çalışmada Yetişkin Van kedilerinde skeleton manus (ossa carpi, ossa metacarpalia I-V ve ossa digitorum manus)'un, bilgisayarlı tomografi görüntülerinden üç boyutlu rekonstrüksiyonu yapılarak anatomi eğitimi açısından kullanılmasının sağlanması, morfolojik olarak incelenmesi, morfometrik ve volümetrik ölçüm değerlerinin elde edilmesi, bu ölçüm değerlerinin cinsiyetler arasındaki biyometrik farklılıkların ortaya konulması amaçlanmıştır.

\section{MATERYAL ve METOT}

Yapılan çalışmada Van Kedisi Araştırma ve Uygulama Merkezi'nden elde edilen 3100- 6000 gr (erkeklerde ortalama ağırlık 4912.5 gr, dişilerde ortalama ağırlık 3600 gr) ağırlığında, 4 ile 6 yaşları arasında toplam 16 adet erişkin Van kedisi (8 dişi, 8 erkek) kullanıldı. Sunulan bu çalışma, Van Yüzüncü Yıl Üniversitesi (Van YYÜ) Hayvan Deneyleri Yerel Etik Kurulu'nun 26.01.2017 ve 2017/01 sayılı kararı ile gerçekleştirildi.

\section{Anestezi}

BT uygulamasına alınan kedilerin anestezisi için, Xylazine (Alfazyne , 1-2 mg/kg) ile Ketamine (Ketasol ${ }^{\circledR}, 15$ mg/kg) kombinasyonu kullanıldı.

\section{Bilgisayarlı Tomografi Görüntülerinin Alınması}

Kedilerin BT tetkikleri için Van YYÜ Tıp Fakültesi Radyoloji Ana Bilim Dalı'nda mevcut 16 dedektörlü çok kesitli BT cihazı (Somatom Sensation 16; Siemens Medical Solutions, Erlangen, Germany) kullanıldı. Anestezi altındaki kediler, prone pozisyonunda simetrik bir şekilde gantri içerisine yerleştirildi. Çekim sırasında BT cihazı parametreleri; kesit kalınlığı, 0.75 $\mathrm{mm}$; fiziksel dedektör collimation, $16 \times 0.6 \mathrm{~mm}$; kernel, U90u; rotation time (sec) değerleri/effective mAs/KV, 0.75/120/120; resolution, $512 \times 512$ pixel; gantry rotation period, $420 \mathrm{~ms}$; final section colimation, $32 \times 0.63 \mathrm{~mm}$; increment, $0.5 \mathrm{~mm}$; feed/rotation, $6 \mathrm{~mm}$ olacak şekilde belirlendi. BT uygulama dozlarının parametreleri literatürler esas alınarak gerçekleştirildi $(10,11)$. Elde edilen imajlar 
DICOM formatında işlenmek üzere iş istasyonuna (Leonardo, Siemens Medical Solutions, Erlangen, Germany) aktarıldı.

\section{Görüntülerin Üç Boyutlu Rekonstrüksiyonu ve Ölçümlerinin Alınması}

Görüntülerin 3 boyutlu (3B) rekonstrüksiyonları iş istasyonunda bulunan Syngo CT software kapsamındaki "volume rendering plus InSpace MPR" yazılımı kullanılarak gerçekleştirildi. 3B görüntü rekonstrüksiyonları $0.75 \mathrm{~mm}$ kalınlıkta oluşturuldu.

\section{Üç Boyutlu Rekonstrüksiyon Görüntülerinin Değerlendirilmesi}

İş istasyonundaki yazılım programı kullanılarak, elde edilen $3 \mathrm{~B}$ rekonstrüksiyon görüntülerindeki yapılar; anatomik, morfometrik ve volümetrik olarak değerlendirildi.

Anatomik Değerlendirme: Üç boyutlu rekonstrüksiyonu gerçekleştirilen ossa carpi, ossa metacarpalia I-V ve ossa digitorum manus'un öncellikle yazılım programı kullanılarak anatomik yapıları belirlendi (Şekil 1). Daha sonra kemikler üzerinde yer alan yapıların, anatomik özellikleri bakımında morfolojik olarak değerlendirilmeleri yapıldı.

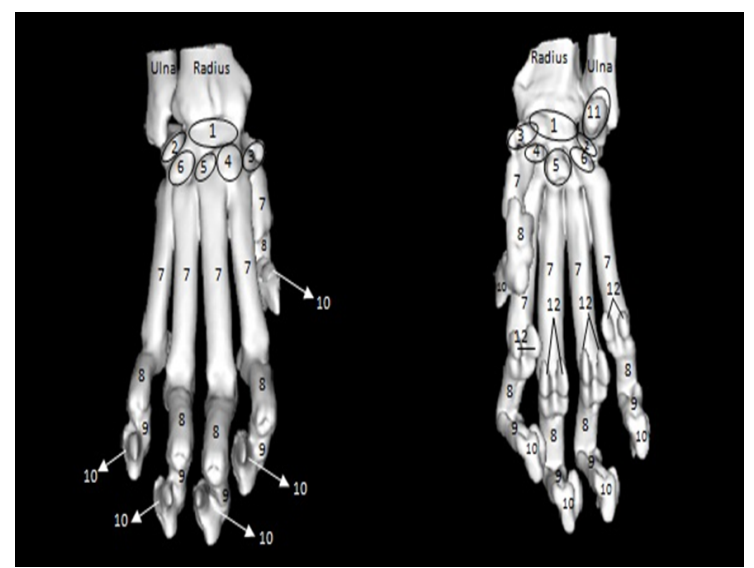

Şekil 1. Van Kedilerinde skeleton manus'un cranial ve caudal'den görünümü. 1- Os carpi intermedioradiale; 2- Os carpi ulnare; 3- Os carpale- I; 4- Os carpale- II; 5- Os carpale- III; 6- Os carpale- IV; 7- Ossa Metacarpalia I-V; 8- Phalanx proximalis; 9- Phalanx media; 10- Phalanx distalis; 11- Os carpi accessorium; 12- Ossa sesamoidea proximalia.

Figure 1. The view of the skeleton manus from cranial and caudal in Van Cats. 1- Os carpi intermedioradiale; 2- Os carpi ulnare; 3- Os carpale- I; 4- Os carpale- II; 5- Os carpale- III; 6- Os carpale- IV; 7- Ossa Metacarpalia I-V; 8- Phalanx proximalis; 9- Phalanx media; 10- Phalanx distalis; 11- Os carpi accessorium; 12- Ossa sesamoidea proximalia.

Tablo 1. Skleton manus'un morfometrik ölçüm noktaları ve kısaltması (cm).

Table 1. Measurement points and abbreviations of the skeleton manus (cm).

\begin{tabular}{ll}
\hline KIsaltma & Açıklama \\
\hline Ossa carpi'nin morfometrik ölçümleri için kullanılan referans noktaları \\
CU & Uzunluk: Ossa carpi'nin proximal ucu ile distal ucu arasındaki mesafe \\
CG & Genişlik: Ossa carpi'nin lateral ucu ile medial ucu arasındaki mesafe \\
CIU & Os carpi intermedioradialis uzunluk: Kemiğin dorso-ventral uzaklığı \\
CIG & Os carpi intermedioradialis genişlik: Kemiğin latero-medial uzaklığı \\
CUU & Os carpi ulnare uzunluk: Kemiğin dorso-ventral uzaklığı \\
CUG & Os carpi ulnare genişlik: Kemiğin latero-medial uzaklığı \\
CAU & Os carpi accessorium uzunluk: Kemiğin dorso-ventral uzaklığı \\
CAG & Os carpi accessorium genişlik: Kemiğin latero-medial uzaklığı \\
CU- I, II, III, IV & Os carpale- I, II, III, IV uzunluk: Kemiklerin dorso-ventral uzaklığı \\
CG-I, II, III, IV & Os carpale- I, II, III, IV genişlik: Kemiklerin latero-medial uzaklığı \\
Ossa metacarpalia I-V'in morfometrik ölçümleri için kullanılan referans noktaları \\
MU-I, II, III, IV & Os metacarpale-I, II, III, IV, V uzunluk: Kemiklerin proximal ucu ile distal ucu arasındaki \\
& mesafe \\
MPLL-I, II, III, IV & Os metacarpale- I, II, III, IV, V proximal L-L çapı: Kemiklerin proximal latero-lateral \\
& uzaklığı \\
MPCC-I, II, III, IV & Os metacarpale- I, II, III, IV, V proximal C-C çapı: Kemiklerin proximal cranio-caudal \\
MMLL-I, II, III, IV & Uzaklığı \\
& Os metacarpale- I, II, III, IV, V medial L-L çapı: Kemiklerin orta noktasının latero-lateral \\
\hline
\end{tabular}


Tablo 1. Skleton manus'un morfometrik ölçüm noktaları ve kısaltması (cm) (DEVAMI).

Table 1. Measurement points and abbreviations of the skeleton manus (cm) (CONTINUE).

\begin{tabular}{|c|c|}
\hline Kisaltma & Açıklama \\
\hline MMCC-I, II, III, IV & $\begin{array}{l}\text { Os metacarpale- I, II, III, IV, V medial C-C çapı: Kemiklerin orta noktasının cranio-caudal } \\
\text { uzaklığı }\end{array}$ \\
\hline MDLL-I, II, III, IV & Os metacarpale- I, II, III, IV, V distal L-L çapı: Kemiklerin distal latero-lateral uzaklığı \\
\hline MDCC-I, II, III, IV & Os metacarpale- I, II, III, IV, V distal C-C çapı: Kemiklerin distal cranio-caudal uzaklığı \\
\hline \multicolumn{2}{|c|}{ Ossa digitorum manus'un morfometrik ölçümleri için kullanılan referans noktaları } \\
\hline PPU-1, 2, 3, 4, 5 & $\begin{array}{l}\text { Phalanx proximalis 1,2,3,4,5 uzunluk: Kemiklerin proximal ucu ile distal ucu arasındaki } \\
\text { mesafe }\end{array}$ \\
\hline PPG-1, 2, 3, 4, 5 & Phalanx proximalis $1,2,3,4,5$ genişlik: Kemiklerin orta noktasının latero-lateral uzaklığı \\
\hline PMU-2, 3, 4, 5 & $\begin{array}{l}\text { Phalanx media 2,3,4,5 uzunluk: Kemiklerin proximal ucu ile distal ucu arasındaki } \\
\text { mesafe }\end{array}$ \\
\hline PMG-2, 3, 4, 5 & Phalanx media 2,3,4,5 genişlik: Kemiklerin orta noktasının latero-lateral uzaklığı \\
\hline PDU-1, 2, 3, 4, 5 & $\begin{array}{l}\text { Phalanx distalis 1,2,3,4,5 uzunluk: Kemiklerin proximal ucu ile distal ucu arasındaki } \\
\text { mesafe }\end{array}$ \\
\hline PDG-1, 2, 3, 4, 5 & Phalanx distalis 1,2,3,4,5 genişlik: Kemiklerin orta noktasının latero-lateral uzaklığı \\
\hline
\end{tabular}

b. Morfometrik Değerlendirme: Anatomik yapıdaki morfometrik ölçümler (uzunluk, çap, en, boy vs.), iş istasyonu üzerindeki görüntülerden ve oluşturulmuş 3B rekonstrüksiyon imajlarından elektronik kaliperler ile yapıldı. Kemikler üzerindeki noktaların morfometrik ölçümleri yapılırken referans noktaları literatür (12) temel alınarak, Tablo 1'de verildi.

c. Volümetrik değerlendirme: Ossa carpi, ossa metacarpalia I-V, ossa digitorum manus'un volüm ölçümlerini hesaplamak amacıyla, iş istasyonu üzerinde aksiyel görüntülerden her bir kemiksel yapı için, kemiğin girdiği bütün kesitler kemik korteksini de içine alacak şekilde çizildi. Çizilen kemik konturları iş istasyonunda bulunan Syngo CT software kapsamındaki "BT volum aplikasyonu (CT volume applications)" aracılığıyla birleştirilerek volümetrik ölçümler elde edildi. Ayrıca, çalışmada kullanılan terminolojik ifadelerde Nomina Anatomica Veterinaria (13) temel alındı.

\section{İstatistiksel Analiz}

Çalışmada, Van kedilerinin ossa carpi, ossa metacarpalia I-V, ossa digitorum manus'un bilgisayarlı tomografiyle üç boyutlu olarak incelenmesi sonucunda elde edilen veriler için tanımlayıcı istatistikler; ortalama, standart sapma, maksimum ve minimum değerler olarak; kategorik değişkenler ise yüzde ve sayı olarak ifade edildi.
Ölçülen değerlerin sürekli değişkenler bakımından grup ortalamalarını karşılaştırmada Mann-Whitney U analizi yapılmıştır. Bu değişkenler arasındaki ilişkiyi belirlemede gruplarda ayrı ayrı olmak üzere Spearman korelasyon katsayıları hesaplanmıştır. Hesaplamalarda istatistik anlamlılık düzeyi \%1 ve \%5 olarak alınmış ve hesaplamalar için SPSS (IBM SPSS for Windows, Ver. 23) istatistik paket programı kullanılmıştır.

\section{BULGULAR}

\section{Ossa carpi'nin Morfolojik, Morfometrik ve Volümetrik Değerlendirilmesi}

Ossa carpi'nin proximal ve distal olmak üzere iki sıradan oluştuğu gözlendi. Proximal'de os carpi intermedioradiale, os carpi ulnare ve os carpi accessorium olmak üzere 3 adet; distal sırada ise os carpale- I, os carpale- II, os carpale- III ve os carpaleIV olmak üzere 4 adet kemik tespit edildi. Bu kemiklerden proximal sıra kemikleri arasında os carpi intermedioradiale'nin en geniş, distal sıra kemikleri arasında ise os carpale-IV'ün en geniş, os carpaleIII'ün en kısa kemik olduğu saptandı.

Cinsiyete göre ossa carpi ölçüm değerleri Tablo 2 'de verildi. Buna göre kedilerdeki ossa carpi'ye ait total volüm, CU, CG, CiU, CiG, CUG, CAU, CAG, CU- I, CG- I, CU- III, CG- III ve CG- IV ölçüm değerlerinin erkek kedilerde dişi kedilere göre istatistiki olarak anlamlı seviyede daha fazla olduğu görüldü $(P<0.05)$. 
Tablo 2. Cinsiyete göre ossa carpi değerlerinin tanımlayıcı istatistikleri ve karşılaştırılması.

Table 2. Descriptive statistics and comparison of the measurements of the ossa carpi by gender.

\begin{tabular}{|c|c|c|c|c|c|c|c|c|c|}
\hline & & \multicolumn{2}{|c|}{ Erkek } & \multicolumn{6}{|c|}{ Dişi } \\
\hline Parametere & Ort. & Std. Sap & Min. & Max. & Ort & Std. Sap & Min. & Max. & $* P$. \\
\hline $\mathrm{CU}$ & 0.91 & 0.03 & 0.88 & 0.95 & 0.80 & 0.08 & 0.68 & 0.90 & 0.001 \\
\hline CG & 1.83 & 0.01 & 1.81 & 1.85 & 1.55 & 0.06 & 1.49 & 1.67 & 0.001 \\
\hline ciu & 0.60 & 0.05 & 0.51 & 0.65 & 0.44 & 0.03 & 0.4 & 0.49 & 0.001 \\
\hline Cig & 0.86 & 0.05 & 0.76 & 0.92 & 0.76 & 0.06 & 0.68 & 0.86 & 0.002 \\
\hline CUU & 0.63 & 0.09 & 0.55 & 0.84 & 0.57 & 0.05 & 0.5 & 0.63 & 0.088 \\
\hline CUG & 0.56 & 0.03 & 0.51 & 0.59 & 0.49 & 0.03 & 0.44 & 0.52 & 0.001 \\
\hline CAU & 0.87 & 0.03 & 0.83 & 0.94 & 0.67 & 0.04 & 0.61 & 0.75 & 0.001 \\
\hline CAG & 0.46 & 0.05 & 0.41 & 0.56 & 0.42 & 0.03 & 0.38 & 0.45 & 0.032 \\
\hline CU-I & 0.74 & 0.03 & 0.69 & 0.8 & 0.64 & 0.06 & 0.52 & 0.72 & 0.001 \\
\hline CG-I & 0.54 & 0.05 & 0.47 & 0.60 & 0.45 & 0.01 & 0.43 & 0.47 & 0.001 \\
\hline CU-II & 0.54 & 0.05 & 0.5 & 0.64 & 0.51 & 0.03 & 0.45 & 0.55 & 0.109 \\
\hline CG-II & 0.45 & 0.05 & 0.35 & 0.50 & 0.43 & 0.03 & 0.39 & 0.47 & 0.176 \\
\hline CU-III & 0.39 & 0.02 & 0.35 & 0.42 & 0.36 & 0.03 & 0.3 & 0.39 & 0.027 \\
\hline CG-III & 0.44 & 0.03 & 0.41 & 0.50 & 0.31 & 0.04 & 0.25 & 0.36 & 0.001 \\
\hline CU-IV & 0.49 & 0.03 & 0.45 & 0.54 & 0.45 & 0.06 & 0.32 & 0.50 & 0.142 \\
\hline CG-IV & 0.64 & 0.04 & 0.58 & 0.68 & 0.59 & 0.05 & 0.48 & 0.63 & 0.035 \\
\hline Total Volüm $\left(\mathrm{cm}^{3}\right)$ & 0.99 & 0.04 & 0.95 & 1.06 & 0.72 & 0.04 & 0.66 & 0.79 & 0.001 \\
\hline
\end{tabular}

\section{Ossa metacarpalia I-V 'in Morfolojik, Morfometrik} ve Volümetrik Değerlendirilmesi

Metacarpal kemiklerin medial'den lateral'e doğru, os metacarpale-I, os metacarpale-II, os metacarpale-III, os metacarpale-IV ve os metacarpale-V şeklinde sıralandığı tespit edildi. Kemiklerin uzunlukları küçükten büyüğe doğru sırasıyla; os metacarpale-I, os metacarpale- $V$, os metacarpale-II, os metacarpale-IV ve os metacarpale-III olduğu tespit edildi. Ayrıca, orta kısımlarının cavum medullare çaplarına bakıldığında bu sıranın, os metacarpale-I, os metacarpale-V, os metacarpale-IV, os metacarpale-II ve os metacarpale-III şeklinde olduğu gözlendi.
Cinsiyete göre os metacarpale I-V ölçüm değerleri Tablo 3'de verildi. Buna göre os metacarpale-I'e ait MU-I, MPLL-I, MMLL-I, MMCC-I, MDLL-I, MDLL-I ve MDCC-I ölçüm değerlerinin; os metacarpale-II’ye ait tüm ölçüm değerlerinin; os metacarpale-III'ye ait MU-III, MPLL-III, MPCC-III, MMCC-III, MDLL-III ve MDCC-III değerlerinin; os metacarpale-IV'ye ait MU-IV, MPLL-IV, MPCC-IV, MMLL-IV, MMCC-IV ve MDLL-IV ölçüm değerlerinin; os metacarpale-V'ye ait MU-V, MPLL-V, MMLL-V, MMCC-V ve MDLL-V ölçüm değerlerinin ve ossa metacarpalia I-V total volüm değerlerinin erkek kedilerde dişi kedilere göre istatistik olarak önemli seviyede daha fazla olduğuna rastlandı $(P<0.05)$. 
Tablo 3. Cinsiyete göre ossa metacarpalia I-V değerlerinin tanımlayıcı istatistikleri ve karşılaştırılması.

Table 3. Descriptive statistics and comparison of the measurements of the ossa metacarpalia I-V by gender.

\begin{tabular}{|c|c|c|c|c|c|c|c|c|c|}
\hline \multirow[b]{2}{*}{ Parametere } & \multirow[b]{2}{*}{ Ort. } & \multicolumn{2}{|c|}{ Erkek } & \multicolumn{6}{|c|}{ Dişi } \\
\hline & & Std. Sap & Min. & Max. & Ort & Std. Sap & Min. & Max. & *P. \\
\hline MU-I & 1.08 & 0.06 & 1.01 & 1.15 & 0.87 & 0.04 & 0.81 & 0.93 & 0.001 \\
\hline MPLL-I & 0.41 & 0.01 & 0.38 & 0.42 & 0.34 & 0.02 & 0.30 & 0.36 & 0.001 \\
\hline MPCC-I & 0.45 & 0.02 & 0.41 & 0.47 & 0.44 & 0.02 & 0.42 & 0.47 & 0.400 \\
\hline MMLL-I & 0.39 & 0.02 & 0.36 & 0.43 & 0.34 & 0.03 & 0.29 & 0.40 & 0.006 \\
\hline MMCC-I & 0.51 & 0.02 & 0.47 & 0.54 & 0.39 & 0.01 & 0.38 & 0.42 & 0.001 \\
\hline MDLL-I & 0.45 & 0.03 & 0.42 & 0.49 & 0.36 & 0.02 & 0.33 & 0.38 & 0.001 \\
\hline MDCC-I & 0.48 & 0.02 & 0.43 & 0.50 & 0.42 & 0.02 & 0.40 & 0.45 & 0.001 \\
\hline MU-II & 2.67 & 0.08 & 2.54 & 2.77 & 2.12 & 0.04 & 2.06 & 2.18 & 0.001 \\
\hline MPLL-II & 0.45 & 0.02 & 0.42 & 0.49 & 0.41 & 0.02 & 0.38 & 0.43 & 0.001 \\
\hline MPCC-II & 0.45 & 0.03 & 0.40 & 0.48 & 0.38 & 0.02 & 0.36 & 0.43 & 0.001 \\
\hline MMLL-II & 0.43 & 0.03 & 0.37 & 0.47 & 0.37 & 0.01 & 0.35 & 0.39 & 0.001 \\
\hline MMCC-II & 0.40 & 0.04 & 0.36 & 0.48 & 0.33 & 0.02 & 0.31 & 0.36 & 0.001 \\
\hline MDLL-II & 0.50 & 0.02 & 0.47 & 0.55 & 0.42 & 0.05 & 0.37 & 0.51 & 0.002 \\
\hline MDCC-II & 0.43 & 0.03 & 0.39 & 0.48 & 0.35 & 0.03 & 0.30 & 0.39 & 0.001 \\
\hline MU-III & 3.20 & 0.14 & 2.98 & 3.40 & 2.66 & 0.10 & 2.53 & 2.82 & 0.001 \\
\hline MPLL-III & 0.48 & 0.03 & 0.41 & 0.51 & 0.38 & 0.08 & 0.28 & 0.49 & 0.004 \\
\hline MPCC-III & 0.43 & 0.03 & 0.39 & 0.47 & 0.36 & 0.04 & 0.30 & 0.42 & 0.002 \\
\hline MMLL-III & 0.42 & 0.01 & 0.40 & 0.43 & 0.40 & 0.04 & 0.33 & 0.44 & 0.138 \\
\hline MMCC-III & 0.40 & 0.03 & 0.38 & 0.46 & 0.37 & 0.02 & 0.34 & 0.39 & 0.014 \\
\hline MDLL-III & 0.52 & 0.01 & 0.50 & 0.54 & 0.39 & 0.03 & 0.34 & 0.42 & 0.001 \\
\hline MDCC-III & 0.41 & 0.02 & 0.38 & 0.46 & 0.36 & 0.03 & 0.31 & 0.40 & 0.003 \\
\hline MU-IV & 2.96 & 0.15 & 2.74 & 3.12 & 2.54 & 0.06 & 2.47 & 2.61 & 0.001 \\
\hline MPLL-IV & 0.39 & 0.03 & 0.36 & 0.43 & 0.33 & 0.02 & 0.30 & 0.36 & 0.001 \\
\hline MPCC-IV & 0.42 & 0.03 & 0.39 & 0.48 & 0.36 & 0.01 & 0.34 & 0.38 & 0.001 \\
\hline MMLL-IV & 0.39 & 0.03 & 0.32 & 0.44 & 0.33 & 0.02 & 0.30 & 0.36 & 0.001 \\
\hline MMCC-IV & 0.43 & 0.02 & 0.40 & 0.45 & 0.39 & 0.04 & 0.35 & 0.46 & 0.034 \\
\hline MDLL-IV & 0.44 & 0.04 & 0.40 & 0.50 & 0.37 & 0.03 & 0.32 & 0.40 & 0.001 \\
\hline MDCC-IV & 0.41 & 0.03 & 0.38 & 0.45 & 0.40 & 0.02 & 0.36 & 0.42 & 0.245 \\
\hline MU-V & 2.54 & 0.10 & 2.41 & 2.68 & 2.07 & 0.06 & 2.00 & 2.15 & 0.001 \\
\hline MPLL-V & 0.39 & 0.02 & 0.34 & 0.41 & 0.33 & 0.02 & 0.31 & 0.35 & 0.001 \\
\hline MPCC-V & 0.49 & 0.03 & 0.45 & 0.52 & 0.46 & 0.03 & 0.40 & 0.49 & 0.052 \\
\hline MMLL-V & 0.39 & 0.02 & 0.34 & 0.41 & 0.33 & 0.02 & 0.31 & 0.35 & 0.001 \\
\hline MMCC-V & 0.49 & 0.05 & 0.41 & 0.55 & 0.40 & 0.02 & 0.38 & 0.44 & 0.001 \\
\hline MDLL-V & 0.45 & 0.02 & 0.41 & 0.48 & 0.38 & 0.02 & 0.36 & 0.41 & 0.001 \\
\hline MDCC-V & 0.42 & 0.03 & 0.38 & 0.46 & 0.41 & 0.02 & 0.36 & 0.42 & 0.310 \\
\hline Total Volüm $\left(\mathrm{cm}^{3}\right)$ & 2.69 & 0.19 & 2.40 & 2.90 & 1.65 & 0.10 & 1.50 & 1.81 & 0.001 \\
\hline
\end{tabular}

* Mann-Whitney U testi sonuçları; P<0.05 olanlar istatistik olarak anlamlı bulunmuştur; Ort.: Ortalama; Std. Sap: Standart Sapma; Min.; Minimum; Max.: Maximum; MU-I, II, III, IV: Os metacarpale-I, II, III, IV, V uzunluk; MPLL-I, II, III, IV: Os metacarpale- I, II, III, IV, V proximal L-L çapı; MPCC-I, II, III, IV: Os metacarpale- I, II, III, IV, V proximal C-C çapı; MMLL-I, II, III, IV: Os metacarpale- I, II, III, IV, V medial L-L çapI; MMCC-I, II, III, IV: Os metacarpale- I, II, III, IV, V medial C-C çapI; MDLL-I, II, III, IV: Os metacarpale- I, II, III, IV, V distal L-L çapı; MDCC-I, II, III, IV: Os metacarpale- I, II, III, IV, V distal C-C çapı.

* Mann-Whitney U test results; P <0.05 were found statistically significant; Mean; Std. Dev: Standard Deviation; Min .; Minimum; Max .: Maximum; MU-I, II, III, IV: Os metacarpale-I, II, III,

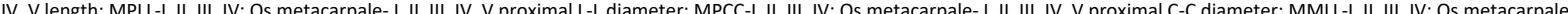

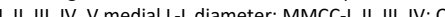
Os metacarpale- I, II, III, IV, V distal C-C diameter.

\section{Ossa digitorum manus'un Morfolojik, Morfometrik} ve Molümetrik Değerlendirilmesi

Ossa digitorum manus'ta toplam beş adet parmak olduğu görüldü. Birinci parmak haricinde, diğer parmak kemiklerinin her biri proximal'den distal'e doğru sırasıyla; phalanx proximalis, phalanx media ve phalanx distalis'ten oluşmaktaydı. Birinci parmakta ise bu kemiklerden phalanx media'nın olmadığı saptandı. Tüm parmak kemiklerinde (birinci parmak hariç) phalanx media'nın, phalanx proximalis'in yarısından daha uzun olduğu gözlendi. Phalanx distalis'in her iki yandan basık olduğu ve uç kısmının tırnak gibi sivrilmiş olduğu tespit edildi. 
Bununla birlikte phalanx distalis'te tuberculum flexorium ve proc. extensorius yapılarının belirgin bir şekilde geliştiği gözlendi. Ayrıca her bir phalanx distalis'in küçük bir tırnakla sonladığı gözlendi.

Cinsiyete göre ossa digitorum manus ölçüm değerleri Tablo 4'te verildi. Buna göre kedilerdeki phalanx proximalis'e ait tüm ölçüm değerlerinin; phalanx media'ya ait PMU-III, PMG-III, PMU-IV ve PMG-V ölçüm değerlerinin; phalanx distalis' ait PDUI, PDG-I, PDU- II, PDG-II, PDG-IV, PDU- V ve PDG-V ölçüm değerlerinin ve ossa digitorum manus total volüm değerlerinin erkek kedilerde dişi kedilere göre istatistik olarak önemli seviyede daha fazla olduğu görüldü $(P<0.05)$.

Tablo 4. Cinsiyete göre ossa digitorum manus değerlerinin tanımlayıcı istatistikleri ve karşılaştırılması.

Table 4. Descriptive statistics and comparison of the measurements of the ossa digitorum manus by gender.

\begin{tabular}{|c|c|c|c|c|c|c|c|c|c|}
\hline & Erkek & & & & Dişi & & & & \\
\hline Parametere & Ort. & Std. Sap & Min. & Max. & Ort & Std. Sap & Min. & Max. & $* P$. \\
\hline PPU-1 & 0.88 & 0.05 & 0.80 & 0.93 & 0.82 & 0.06 & 0.73 & 0.88 & 0.031 \\
\hline PPG-1 & 0.43 & 0.04 & 0.38 & 0.51 & 0.37 & 0.02 & 0.35 & 0.39 & 0.002 \\
\hline PPU-2 & 1.21 & 0.12 & 1.02 & 1.38 & 1.08 & 0.08 & 0.98 & 1.20 & 0.023 \\
\hline PPG-2 & 0.49 & 0.04 & 0.41 & 0.53 & 0.40 & 0.03 & 0.37 & 0.45 & 0.001 \\
\hline PPU-3 & 1.34 & 0.16 & 1.12 & 1.63 & 1.14 & 0.04 & 1.06 & 1.20 & 0.004 \\
\hline PPG-3 & 0.47 & 0.03 & 0.42 & 0.51 & 0.42 & 0.02 & 0.40 & 0.45 & 0.001 \\
\hline PPU-4 & 1.40 & 0.06 & 1.28 & 1.49 & 1.16 & 0.04 & 1.10 & 1.21 & 0.001 \\
\hline PPG-4 & 0.42 & 0.03 & 0.38 & 0.47 & 0.36 & 0.02 & 0.34 & 0.39 & 0.001 \\
\hline PPU-5 & 1.13 & 0.04 & 1.08 & 1.20 & 1.00 & 0.04 & 0.93 & 1.03 & 0.001 \\
\hline PPG-5 & 0.41 & 0.03 & 0.36 & 0.47 & 0.36 & 0.01 & 0.34 & 0.37 & 0.001 \\
\hline PMU-2 & 0.78 & 0.09 & 0.65 & 0.90 & 0.72 & 0.06 & 0.60 & 0.80 & 0.183 \\
\hline PMG-2 & 0.38 & 0.04 & 0.31 & 0.42 & 0.37 & 0.02 & 0.35 & 0.40 & 0.329 \\
\hline PMU-3 & 0.94 & 0.10 & 0.82 & 1.10 & 0.75 & 0.03 & 0.71 & 0.81 & 0.001 \\
\hline PMG-3 & 0.42 & 0.03 & 0.35 & 0.44 & 0.31 & 0.02 & 0.28 & 0.34 & 0.001 \\
\hline PMU-4 & 0.92 & 0.09 & 0.84 & 1.08 & 0.67 & 0.05 & 0.62 & 0.75 & 0.001 \\
\hline PMG-4 & 0.41 & 0.06 & 0.33 & 0.50 & 0.37 & 0.01 & 0.35 & 0.38 & 0.053 \\
\hline PMU-5 & 0.86 & 0.12 & 0.73 & 1.00 & 0.86 & 0.04 & 0.80 & 0.90 & 0.889 \\
\hline PMG-5 & 0.40 & 0.05 & 0.30 & 0.46 & 0.31 & 0.01 & 0.30 & 0.33 & 0.001 \\
\hline PDU-1 & 0.76 & 0.06 & 0.68 & 0.86 & 0.60 & 0.03 & 0.56 & 0.63 & 0.001 \\
\hline PDG-1 & 0.67 & 0.05 & 0.61 & 0.75 & 0.49 & 0.03 & 0.43 & 0.54 & 0.001 \\
\hline PDU-2 & 0.78 & 0.07 & 0.68 & 0.89 & 0.68 & 0.05 & 0.62 & 0.77 & 0.004 \\
\hline PDG-2 & 0.62 & 0.05 & 0.57 & 0.70 & 0.51 & 0.05 & 0.42 & 0.57 & 0.001 \\
\hline PDU-3 & 0.74 & 0.05 & 0.68 & 0.80 & 0.69 & 0.07 & 0.58 & 0.78 & 0.155 \\
\hline PDG-3 & 0.60 & 0.06 & 0.51 & 0.67 & 0.57 & 0.04 & 0.49 & 0.60 & 0.309 \\
\hline PDU-4 & 0.83 & 0.08 & 0.71 & 0.93 & 0.82 & 0.05 & 0.76 & 0.91 & 0.672 \\
\hline PDG-4 & 0.73 & 0.03 & 0.68 & 0.79 & 0.50 & 0.07 & 0.42 & 0.61 & 0.001 \\
\hline PDU-5 & 0.84 & 0.03 & 0.81 & 0.87 & 0.77 & 0.03 & 0.74 & 0.81 & 0.001 \\
\hline PDG-5 & 0.74 & 0.05 & 0.63 & 0.80 & 0.58 & 0.04 & 0.52 & 0.62 & 0.001 \\
\hline Total Volüm $\left(\mathrm{cm}^{3}\right)$ & 2.58 & 0.20 & 2.24 & 2.77 & 1.49 & 0.11 & 1.33 & 1.65 & 0.001 \\
\hline
\end{tabular}




\section{TARTIŞMA ve SONUÇ}

Yapılan çalışmada ossa carpi'nin proximal ve distal olmak üzere iki sıradan oluştuğu, proximal'de os carpi radiale ile os carpi intermedium'un kaynaşarak oluşturduğu os carpi intermedioradiale, os carpi ulnare ve os carpi accessorium olmak üzere 3 adet; distal sırada ise os carpale- I, os carpale- II, os carpale- III ve os carpale- IV olmak üzere 4 adet kemik bulunduğu tespit edilmiştir. Dursun (2), Liebich ve ark. (4), Dyce ve ark. (3), Bahadır ve Yıldız (5), skeleton manus'taki bu kemik sıralarının evcil carnivor'larda da benzer olduğunu bildirmişlerdir. Aynı şekilde Özkan (14), kirpilerde proximal sırada 3, distal sırada 4 adet kemik bulunduğunu, bu kemiklerden proximal sıra kemikleri arasında os carpi intermedioradiale'nin, distal sıra kemikleri arasında ise os carpale-IV'ün en geniş kemik olduğunu bildirmiştir. Kirpi ossa carpi'sinin bu özelliklerinin çalışma verileriyle uyum içerisinde olduğu görülmüştür. Ayrıca, Atalar ve Karan (15), sansarlarda da proximal sırada 3 adet, distal sırada 4 adet ossa carpi'nin bulunduğunu bildirmiştir. Bununla birlikte, Yılmaz ve ark. (16) oklu kirpilerde ve Karan (17) yaban domuzlarında proximal sırada 4 adet, distal sırada 4 adet; Tobechukwu (18), grascutterlerde proximal sırada 3 adet, distal sırada 5 adet; Saber (19), wombatlarda proximal sırada 4 adet; distal sırada 2 adet ve Özkan (20), mole-ratlarında proximal sırada 2 adet, distal sırada 4 adet ossa carpi'nin olduğunu bildirmişlerdir. Oklu kirpi, yaban domuzu, grasscutter, wombat ve mole-rat ossa carpi'lerinde görülen bu kemik sayıları çalışma bulgularını desteklememektedir. Ayrıca Özkan (20) moleratlarda ve Dursun (2) tavşanlarda proximal ve distal sıralara arasında os carpi centrale'nin bulunduğunu bildirmişlerdir. Yapılan çalışmada böyle bir bulguya rastlanılmamıştır.

Girgin ve ark. (21) tilki, kurt ve yerli köpeklerde, Yılmaz ve ark. (16) oklu kirpilerde, Özkan (14) kirpilerde, Atalar ve Karan (15) sansarda ve Özkan (20) mole-ratlarında 5 tane metacarpal kemiğin bulunduğunu ve bu kemiklerden ise os metacarpaleIII'ün en uzun, os metacarpale-I'in ise en kısa kemik olduğunu rapor etmişlerdir. Karan ve Atalar (22) sincaplarda, Karan (17) yaban domuzlarında ve Gough-Palmer (23) mirketlerde os metacarpale-l'in bulunmadığından dolayı 4 adet metacarpal kemiğin bulunduğunu bildirmişlerdir. Araştırmada, 5 tane metacarpal kemiğin bulunduğu ve bu kemiklerin uzunluklarının küçükten büyüğe doğru sırasıyla; os metacarpale-I, os metacarpale-V, os metacarpale-II, os metacarpale-IV ve os metacarpale-III olduğu tespit edilmiştir.

Yapılan araştırmada ossa digitorum manus'un 5 tane parmaktan oluştuğu, sadece birinci parmakta phalanx media'nın olmadığı gözlenmiş olup, bu bulguların literatür verileriyle aynı şekilde olduğu tespit edilmiştir (2-4).

Atalar ve Karan (15) sansarlarda, Karan ve Atalar (22) sincaplarda, Yılmaz ve ark. (16) oklu kirpilerde phalanx media'nın phalanx proximalis'in yaklaşık yarısı kadar olduğunu bildirmelerine rağmen, yapılan çalışmada phalanx media'nın, phalanx proximalis'in yarısından daha uzun olduğu gözlenmiştir.

Dursun (2) evcil Carnivor'larda, Yılmaz ve ark. (16) oklu kirpilerde phalanx distalis'in her iki yandan basık olduğunu, uç kısmının tırnak gibi sivrildiğini, proc. extensorius, tuberculum flexorium ve tuberculum flexorium'un yanlarında for. soleare yapılarının bulunduğunu bildirmişlerdir. Araştırmada, bu bulgulardan tuberculum flexorium'un yanlarında bulunan for. soleare yapısına rastlanılmamıştır. Ayrıca phalanx distalis'in Bahadır ve Yıldız (5) evcil carnivor'larda, Yılmaz ve ark. (24) su samurlarında, Karan ve Atalar (22) sincaplarda, Atalar ve Karan (15) sansarlarda küçük bir tırnağa sahip olduğunu, Atalar ve Karan (15) porsuklarda ise phalax distalis'in oldukça büyük bir tırnak taşıdığını bildirmişlerdir. Yapılan çalışmada ise phalanx distalis'in evcil carnivor, su samuru, sincap ve sansarlarda olduğu gibi küçük bir tırnakla sonladığı görülmüştür.

Lokomosyon, hayvan ekolojisinde oldukça önemli bir yer tutmaktadır (25). Skeleton manus ise, tüm canlılarda lokomosyonun önemli bir parçasını oluşturmaktadır (26). Bununla birlikte skeleton manus, özellikle kedi gibi carnivor hayvanlarda 
avlarını yakalama-tutma, tırmanma, pençeleme, koşma, hızlanma gibi davranışsal hareketlerin meydana getirilmesinde oldukça önemli bir yer tutmaktadır $(23,25,26)$. Bu duruma paralel olarak yapılan çalışmada Van kedilerinde skeleton manus ayrıntılı bir şekilde morfolojik, morfometrik ve volümetrik olarak incelenmiştir. Bu ölçümlerde skeleton manus kemiklerinin L-M genişliği özellikle dikkat çekmektedir. Bu durum diğer carnivor'larda olduğu gibi avlarını yakalama-tutma, tırmanma, pençeleme, koşma ve hızlanma gibi davranışsal hareketlerin Van kedilerinde de iyi bir şekilde geliştiğini göstermektedir. Bununla birlikte skeleton manus'u oluşturan kemiklerin L-M genişlemesi Van kedilerinde el bölgesinin genişlemesine neden olmaktadır. Ayrıca bu anatomik özelliklerinden dolayı yüzme sporunu diğer kedilerden daha çok seven ve bu sporu kolaylıkla yapan Van kedilerinde, yüzme ve kulaç atma işleminin daha kolay yapıldığı düşünülmektedir.

Bilgisayarlı tomografi ve üç boyutlu yazılım programları, ilgili anatomik yapıdaki morfometrik ölçüm değerlerinin yanı sıra volümetrik ölçüm değerlerinin de elde edilmesine olanak sağlamaktadır $(27,28)$. Bu amaçla yapılan çalışmada Van kedilerinin ön bacak iskeletini oluşturan kemiklere ait volümetrik ölçüm değerleri elde edilmiştir. Cinsiyete göre bu volüm değerlerine bakıldığında, kedilerdeki ossa carpi, ossa metacarpalia I-V ve ossa digitorum manus ölçüm değerlerinin erkek kedilerde dişi kedilere göre istatistik olarak anlamlı seviyede daha yüksek olduğu tespit edildi.

Sonuç olarak, Van kedilerinde skeleton manus volümetrik, morfometrik ve morfolojik olarak değerlendirildi. Bu kemiklerin osteometrik ölçüm değerlerinin istatistiksel olarak cinsiyetler arasındaki biyometrik farklılıkları belirlendi. Ayrıca bu çalışmanın, Van kedilerinde iskelet sistemi ile ilgili anatomi eğitimi başta olmak üzere, cerrahi ve klinik uygulama alanlarında veteriner hekimlere ve eğitim alan öğrencilere faydalı olabileceği kanısına varılmıştır.

\section{Çıkar Çatışması}

Yazarlar, çıkar çatışması olmadığını beyan eder.

\section{KAYNAKLAR}

1. Cak B., 2017. Turkish Van cat and Turkish Angora cat: a review. J Agr Sci Tech A, 7, 3, 151-159.

2. Dursun N., 2008. Veteriner Anatomi I. Medisan Yayınevi, Ankara.

3. Dyce KM., Sack WO., Wensing CJG., 2002. Textbook of Veterinary Anatomy. 3rd ed., Saunders, Philadelphia, United States.

4. Liebich HG., Maierl J., König HE., 2015. Ön bacaklar (membra thoracica). In "Veteriner Anatomi (Evcil Memeli Hayvanlar)”. Ed., HE König, HG Liebich (Ed), Çev Ed., i Kürtül, i Türkmenoğlu, 6th ed., 151-222, Medipres, Malatya.

5. Bahadır A., Yıldız H., 2008. Veteriner Anatomi: Hareket Sistemi \& İç Organlar. Ezgi Kitabevi, Bursa.

6. Brenton H., Hernandez J., Bello F., Strutton P., Purkayastha S., Firth T., Darzi A., 2007. Using multimedia and web 3D to enhance anatomy teaching. Comput Educ, 49, 32-53.

7. Özkadif S., Haligur A., Eken E., 2019. A threedimensional reconstruction of the scapula in the red fox (Vulpes vulpes). Indian J Anim Res, 53, 336-340.

8. Ohlerth S., Scharf G., 2007. Computed tomography in small animals-basic principles and state of the art applications. Vet J, 173, 25471.

9. Yılmaz O., Soyguder Z., Yavuz A. 2020. Threedimensional investigation by computed tomography of the clavicle and scapula in Van cats. Van Vet J, 31, 1, 34-41.

10. Prokop M., 2003. General principles of MDCT. Eur J Radiol, 45, 4-10.

11. Kalra MK., Maher MM., Toth TL., Hamberg LM., Blake MA., Shepard J., Saini S., 2004. Strategies for CT radiation dose optimization. Radiology, 230, 619-628.

12. Von Den Driesch A., 1976. A guide to the 
measurement of animal bones from archaeological sites. Peabody Museum Bulletins, Harvard University, The United States of America.

13. Nomina Anatomica Veterinaria., 2017. Prepared by the international committes on veterinary gross anatomical nomenclature and authorized by the general assambly of the world association of veterinary anatomists. 6th Ed., the editorial committee Hanover (Germany), Ghent (Belgium), Columbia, MO (U.S.A.), Rio de Janeiro (Brazil).

14. Özkan ZE., 2004. Macro-anatomical investigations on the Hedgehog skeleton (Erinaceus europaeus) I- Ossa membri thoracici. Turk J Vet Anim Sci, 28, 271-274.

15. Atalar Ö., Karan M., 2002. Macro-anatomical investigations on the skeletons of Marten (Martes foina) I. Ossa membri thoracici. FÜ Sağlık Bil Dergisi, 16, 2, 229-232.

16. Yılmaz S., Özkan ZE., Özdemir D., 1998. Macroanatomical investigations on the skeletons of Porcupine (Hystrix cristata) I. Ossa membri thoracici. Turk J Vet Anim Sci, 22, 389-392.

17. Karan M., 2012. Macro-anatomical study of ossa membri thoracici in the Feral pigs (Sus scrofa). FÜ Sağ Bil Vet Derg, 26, 1, 17-20.

18. Tobechukwu OK., Adeniyi OS., Olajide HJ., Tavershima D., Sulaiman SO., 2015. Macroanatomical and morphometric studies of the Grasscutter (Thryonomyss winderianus) forelimb skeleton. Int J Vet Sci Anim Husb, 2, 006-012.

19. Saber AS., 2013. Some morphological observations on the thoracic limb bones of the Hairy-Nosed Wombat (Lasiorhinus latifornis, Owen). J Vet Anat, 6, 93-109.

20. Özkan ZE., 2002. Macro-anatomical investigations on the forelimb skeleton of MoleRat (Spalax leucodon nordmann). Vet Arhiv, 72, 91-99.

21. Girgin A., Karadağ H., Bilgiç S., Temizer A., 1988. A study on the macro-anatomical differences of the skeletons of wolf and fox as compared with the skeleton of dog. Sü Vet Fak Derg, 4, 169-182.

22. Karan M., Atalar Ö., 2003. Macro-anatomical investigations on the skeletons of squirrels (Sciurus vulgaris) I. Ossa membri thoracici. FÜ Sağlık Bil Derg, 17, 35-38.

23. Gough-Palmer AL., Maclachlan J., Routh A., 2008. Paws for thought: comparative radiologic anatomy of the mammalian forelimb. Radio Graphics, 28, 501-510.

24. Yılmaz S., Dinç G., Özdemir D., 1999. Su samuru (Lutra lutra) iskelet sistemi üzerinde makroanatomik araştırmalar. I. Ossa membri thoracici. FÜ Sağlık Bil Derg, 13, 225-228.

25. Martin-Serra A., Figueirido B., Palmqvist P., 2014. A three-dimensional analysis of morphological evolution and locomotor performance of the carnivoran forelimb. Plos One, 9, 1-20.

26. Meachen-Samuels J., Valkenburgh BV., 2009. Forelimb indicators of prey-size preference in the felidae. J Morphol, 270, 729-744.

27. Yılmaz O., 2018. Van kedilerinde ön bacak iskeletinin bilgisayarlı tomografi ile üç boyutlu olarak incelenmesi. Van Yüzüncü Yıl Üniversitesi, Sağlık Bilimleri Enstitüsü, Türkiye.

28. Yilmaz O., Soyguder Z., Yavuz A., Dundar I., 2020. Three-dimensional computed tomographic examination of pelvic cavity in Van cats and its morphometric investigation. Anat Histol Embryol, 49, 60-66. 